\title{
A New Implant for Transfemoral Amputation: Improved Gait and Comfort
}

\author{
Mathieu Assal ${ }^{1,2}$, Halah Kutaish ${ }^{1,2 *}$, Richard Stern ${ }^{1}$, Alice Bonnefoy-Mazure ${ }^{3}$, Antoine Acker ${ }^{1}$, \\ Axel Gamulin4, Stephane Armand 3 , Gorki Carmona 3 , Alain Lacraz ${ }^{4}$
}

${ }^{1}$ Foot \& Ankle Surgery Center, Centre Assal, Hirslanden Clinique La Colline, Geneva, Switzerland

${ }^{2}$ Faculty of Medicine, Geneva University, Geneva, Switzerland

${ }^{3}$ Willy Taillard Laboratory of Kinesiology, Geneva University Hospitals and Geneva University, Geneva, Switzerland

${ }^{4}$ Division of Orthopaedic Surgery and Traumatology, University Hospitals of Geneva, Geneva, Switzerland

Email: ^hala.kutaish@gmailcom

How to cite this paper: Assal, M., Kutaish, H., Stern, R., Bonnefoy-Mazure, A., Acker, A., Gamulin, A., Armand, S., Carmona, G. and Lacraz, A. (2021) A New Implant for Transfemoral Amputation: Improved Gait and Comfort. Open Journal of Orthopedics, 11, 199-205. https://doi.org/10.4236/ojo.2021.116019

Received: May 5, 2021

Accepted: June 26, 2021

Published: June 29, 2021

Copyright $\odot 2021$ by author(s) and Scientific Research Publishing Inc. This work is licensed under the Creative Commons Attribution International License (CC BY 4.0).

http://creativecommons.org/licenses/by/4.0/ (c) (i) Open Access

\begin{abstract}
Introduction: Transfemoral amputation results in a prosthesis which bears weight on the ischium. Gait disturbance, lack of an end-bearing stump and discomfort in the groin from the socket even while sitting, are important issues. Methods: This is a pilot report of an ongoing randomized blind clinical trial of a new intramedullary implant post transfemoral amputation. Here, we describe a single case illustrating the surgical technique and clinical outcome of a dysfunctional post-traumatic transfemoral amputation addressed with this implant. Clinical gait analysis, SF-12 and VAS were assessed pre- and post-intervention at 6 months of follow-up. Results: An improved stump control is accomplished by means of myoplasty and myodesis through an end-cap. Stride width improved from $0.21 \mathrm{~m}$ pre-op to $0.13 \mathrm{~m}$ post-op, and more symmetrical stride length ( $\Delta 0.21 \mathrm{~m}$ pre-op vs. $\Delta 0.06 \mathrm{~m}$ post-op) was noted, indicating improved gait quality and stability. Gait velocity increased $(0.51 \pm 0.04 \mathrm{~m} / \mathrm{s}$ pre-op vs. 0.64 $\pm 0.02 \mathrm{~m} / \mathrm{s}$ post-op). Conclusion: This technique reveals improvements in gait parameters in a transfemoral amputee treated with a new procedure. Improved prosthesis control, sitting comfort, greater hip range of motion, better gait stability, and enhanced walking abilities were noted.
\end{abstract}

\section{Keywords}

Above Knee Amputation, Transfemoral Amputation, Gritti-Stokes, Weight-Bearing End-Cap

\section{Main Text}

\subsection{Background}

In 1857 Rocco Gritti, a young surgeon at Ospedale Maggiore in Milan published 
an article describing a method of supracondylar femoral amputation using the patella as an osteoplastic flap to provide an end-bearing stump [1]. The procedure was modified by Stokes [2] and is known as Gritti-Stokes procedure. It remains in widespread use today, most often for amputations secondary to vascular disease, as well as in trauma [3].

Gritti's concept is that the level of transfemoral amputation is of prime importance for success of the procedure [4] [5]. The level should be low to allow the patella to fuse on a wide area of cancellous bone. Gritti recommended that the femur should be transected at the upper level of the old epiphyseal growth plate, corresponding to the adductor tubercle. Its use is restricted to cases where the amputation level is just above the femoral condyles. The possible benefit of an end-bearing stump as compared to a standard above-knee amputation is an easier prosthetic fitting since the pelvis is free of contact from the prosthesis; this observation has been reported by Faber et al. [6]. In addition, a decreased need for assistive devices for mobilization and wheelchair use have been described by Taylor et al. [3].

This short pilot report describes a novel intramedullary implant currently being studied by an ongoing randomized blind clinical trial in the context of a transfemoral amputation. The implant used is a femoral stem (MAKAN ${ }^{\oplus}$; Medacta International, Castel San Pietro, Switzerland) cemented into the proximal femur and attached to a distal end-cap. This system allows for end-bearing on the end-cap even with more proximal transfemoral amputation and permits to bypass ischial bearing.

\subsection{Case Presentation}

A 60-year-old man presented 4 years post motorcycle accident which resulted in a transfemoral amputation at the junction of the middle and distal thirds of the left femur. The traumatic transfemoral amputation was treated by the trauma team utilizing a standard surgical technique including wound debridement, myodesis and myoplasty, and primary closure. At the same time, he sustained an ipsilateral pertrochanteric fracture which was fixed with a sliding hip screw. Both interventions healed uneventfully.

During physical rehabilitation, the patient reported moderate to severe discomfort at the ischium as well as substantial difficulties in gait requiring constant use of two crutches. This was despite several attempts to improve prosthetic fitting as well as extensive physical therapy. Examination revealed a dysfunctional amputation with a miss-match between the tissue stump and the bone stump due to tissue redundancy. The prosthesis had a vacuum suspension socket, integrated ischium support, C-Leg knee and C-Walk foot (Ottobock ${ }^{\odot}$, Ottobock SE \& Co. KGaA).

After institutional board approval and signed informed consent, the patient was offered a revision of the stump with the $\mathrm{MAKAN}^{\circledR}$ implant (Figure 1).

\subsection{Implant Description}

The MAKAN implant consists of a cemented intramedullary stem attached to a 


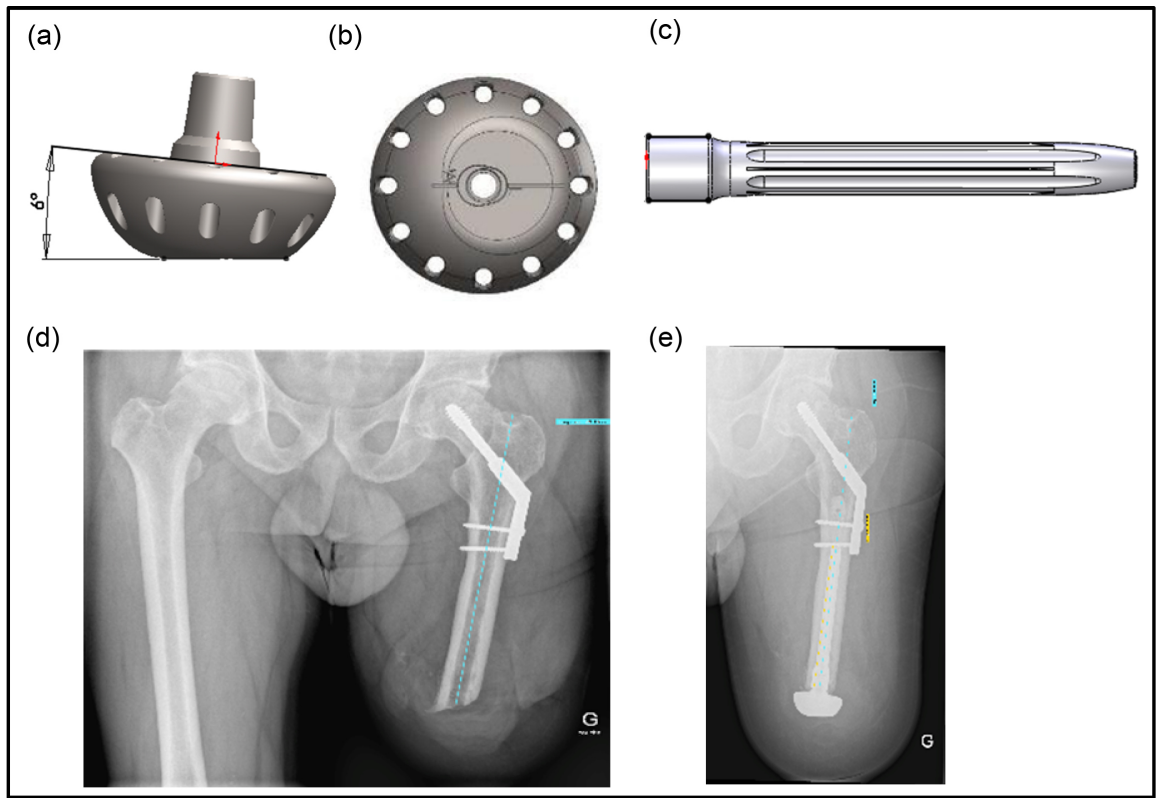

Figure 1. Top panel: The MAKAN ${ }^{\circledR}$ implant, (a) coronal view of the cap; (b) axial view of the cap from below; (c) intramedullary stem. Bottom panel: (d), (e) Pre- and post-operative standard radiographs showing implant position.

distal end-cap (Figures 1(a)-(c)). The intramedullary component is the femoral stem of a total knee arthroplasty (GMK Revision, Medacta International, Castel San Pietro, Switzerland). The end cap is intimately attached to the femoral stem through a press-fit mechanism and secured with an axial compression screw. The cap comes in various sizes with a 6-degree valgus wedge to restore the mechanical axis of the femur. This implant obtained a CE certification (Reg. No: MED 29022-B).

\subsection{Surgical Procedure}

Pre-operative radiographs and computed tomography were obtained to determine implant length and diameter.

The patient underwent surgery in the supine position and a sterile thigh pneumatic tourniquet was applied. The skin incision was made through the previous surgical scar down to the tip of the remaining femur. The various muscle compartments were identified and prepared for later myoplasty and myodesis. The intramedullary canal was visualized and prepared for reaming. Sequential reamers from $11 \mathrm{~mm}-16 \mathrm{~mm}$ were used to prepare the intramedullary canal up to the planned implant size. A16mm diameter $\mathrm{MAKAN}^{\circledR}$ stem was cemented into the medullary canal, as the implant addresses different levels of amputation where the intramedullary canal maybe too wide. Then a medium size cap was press-fitted to the stem and secured with an axial screw. The size of the cap was selected as to accurately match the surface of the patella according to Gritti's principles. To secure the various muscles, particularly the hamstrings, myoplasty and myodesis reconstruction of the stump was carried out with FiberWire (Arthrex, Switzerland) inserted through the multiple holes in the cap. After irri- 
gation and hemostasis, the stump was closed in layers and loosely dressed. A post-operative radiograph was obtained (Figure 1(d) and Figure 1(e)).

Once the wound was healed the patient was fitted with a new prosthesis which included a silicone liner, vacuum suspension and a custom-made hard socket without any pelvic or ischial contact. The C-Leg knee and C-Walk foot (Ottobock $^{\circledR}$, Ottobock SE \& Co. KGaA, Duderstadt, Germany) were maintained.

In this setting, load transfer was assumed to occur at the distal stump and at the level of the soft tissues surrounding the femur.

\subsection{Outcome Measures}

The choice of the outcome measurements was based on the recommendation of Esquenazi et al. 2014 in "Gait analysis in Lower-Limb Amputation and Prosthetic Rehabilitation" [7]. These included Clinical gait analysis (CGA) using a 12-camera motion analysis system (Oqus 7+, Qualisys, Gothenburg, Sweden). Markers were placed on the lower limbs and pelvis according to the Conventional Gait Model [8]. Data was collected for at least five gait cycles. Visual 3D (C-Motion, Inc., Germantown, USA), the open-source Biomechanical ToolKit package [9] and Matlab R2012b (MathWorks, Natick, USA) were used for kinematics, data analysis, and gait quality scores calculation (Figure 2 and Table 1). Stride width, stride length and gait velocity were measured. Gait stability and walking ability were assessed with the Tinetti test [10] and the Gillette Gait Index (GGI) [11]. Physical function was assessed through the 6-minute Walk Test

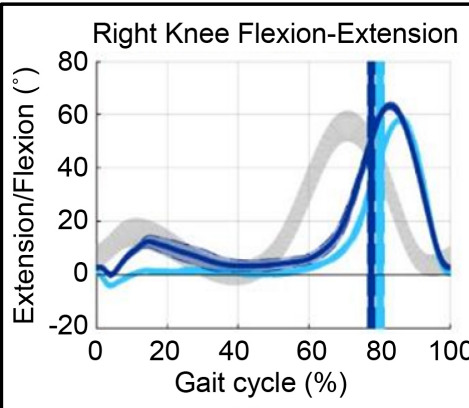

(a)

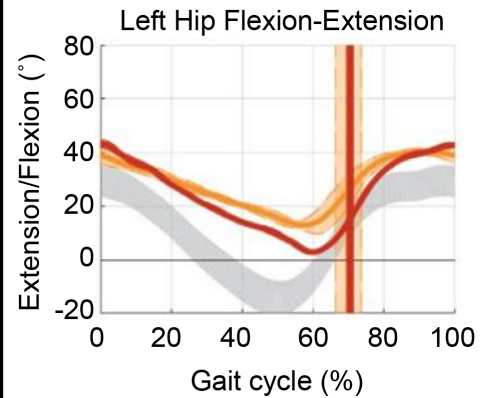

(c)

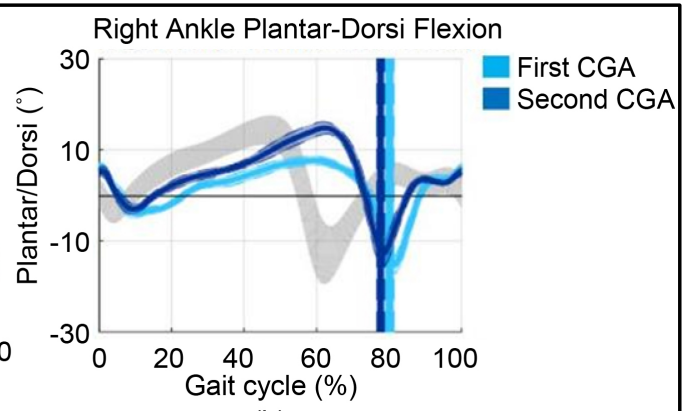

(b)

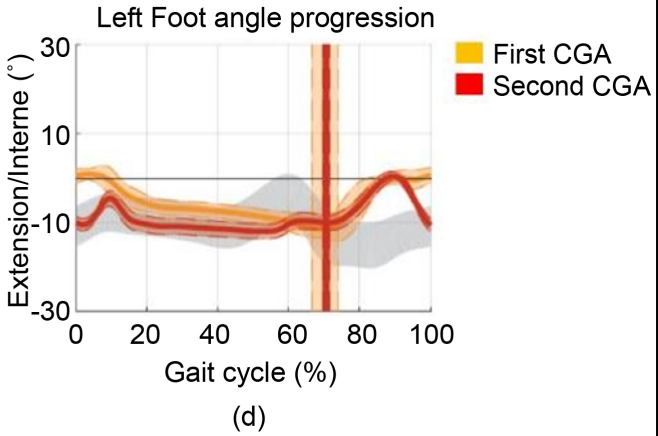

Figure 2. Kinematic curves before and after surgery: (a) right knee, (b) right ankle, (c) left hip, and (d) left foot. First CGA performed before the MAKAN ${ }^{\circ}$ implant, second CGA performed at 6 months post implant. Grey area is the healthy gait $+/-$ one standard deviation. Vertical lines are foot-offs. 
Table 1. Spatio-temporal parameters (i.e., gait velocity, cadence, percentage of stance phase, stride length, and step length) at each visit. The operated and non-operated sides were analysed and compared for walking trial and kinematic curves in the sagittal plane, as well as the range of motion for hip, knee and ankle joints.

\begin{tabular}{|c|c|c|}
\hline & $\begin{array}{l}\text { Before Surgery } \\
\text { Mean (SD) }\end{array}$ & $\begin{array}{c}\text { After Surgery } \\
\text { Mean (SD) }\end{array}$ \\
\hline \multicolumn{3}{|l|}{ Spatio-Temporal } \\
\hline Gait Velocity (m/s) & $0.51(0.04)$ & $0.64(0.02)$ \\
\hline Cadence (steps/min) & $71.87(2.53)$ & $74.36(1.25)$ \\
\hline \multicolumn{3}{|l|}{ Stance phase (\%) } \\
\hline Operated limb & $70.1(3.7)$ & $70.6(0.89)$ \\
\hline Non-operated $\operatorname{limb}$ & $80.1(1.1)$ & $77.7(0.96)$ \\
\hline \multicolumn{3}{|l|}{ Stride length (m) } \\
\hline Operated limb & $0.54(0.02)$ & $0.55(0.03)$ \\
\hline Non-operated limb & $0.33(0.02)$ & $0.49(0.01)$ \\
\hline Step width (m) & $0.21(0.01)$ & $0.13(0.01)$ \\
\hline \multicolumn{3}{|l|}{ Kinematics } \\
\hline \multicolumn{3}{|c|}{ Hip Flexion range - gait cycle $\left({ }^{\circ}\right)$} \\
\hline Operated limb & $27.4(5.5)$ & $40.6(1.5)$ \\
\hline Non-operated limb & $40.7(1.5)$ & $42.7(1.7)$ \\
\hline \multicolumn{3}{|c|}{$\begin{array}{l}\text { Knee Flexion range - gait cycle } \\
\left({ }^{\circ}\right)\end{array}$} \\
\hline Operated limb & $50.8(14.6)$ & $52.8(4.1)$ \\
\hline Non-operated limb & $62.8(3.2)$ & $63.6(2.9)$ \\
\hline \multicolumn{3}{|c|}{$\begin{array}{l}\text { Ankle dorsiflexion range - gait } \\
\text { cycle }\left({ }^{\circ}\right)\end{array}$} \\
\hline Operated limb & $8.6(2.3)$ & $10.3(0.9)$ \\
\hline Non-operated limb & $23.0(2.2)$ & $26.7(2.8)$ \\
\hline
\end{tabular}

(6-WT). Short Physical Performance Battery (SPPB) and Timed Up \& Go (TUG) were computed. Quality of life was assessed by Short Form-12 (SF-12). Level of pain was evaluated with the Visual Analogue Scale (VAS) and comfort was discussed with the patient at each visit. These data were measured pre-operatively and at 6 months post-operatively.

\section{Results and Discussion}

Results showed improved stride width $(0.21 \mathrm{~m}$ pre-op vs. $0.13 \mathrm{~m}$ post-op), and a more symmetrical stride length ( $\Delta 0.06 \mathrm{~m}$ post-op vs. $\Delta 0.21 \mathrm{~m}$ pre-op) indicating improved gait quality and stability. Gait velocity increased $(0.64 \pm 0.02 \mathrm{~m} / \mathrm{s}$ vs. $0.51 \pm 0.04 \mathrm{~m} / \mathrm{s})($ Table 1$)$.

The Tinetti test evolved from 22 pre-op to 25 points post-op, indicating improved stability. GGI evolved on the left side from 87 pre-op to 94 post-op and on the right side from 148 pre-op to 120 post-op respectively indicating overall gait improvement. Ankle and knee kinematics improved on the sound side 
(Figure 2(a) and Figure 2(b)). Furthermore, a better angle of foot progression and hip kinematics were noted (Figure 2(c) and Figure 2(d)). The 6-WT improved by $31.5 \%$ ( $165 \mathrm{~m}$ pre-op to $240 \mathrm{~m}$ post-op). The SPPB went from 7 pre-op to 9 points post-op (range 0 - 12 points, 12 indicates the highest degree of lower extremity function). TUG was performed in 18 seconds instead of 23 with traditional hard socket reflecting a better balance and stability.

The scores in physical (PCS-12) and mental (MCS-12) components of SF-12 increased from PCS-12 23.4/MSC-12 47.6 to PCS-12 46.1/MSC-12 62.4. The pain score VAS went from 4 to 2 . The patient described increased comfort while sitting as noted by a reduced need for changing positions, absence of groin irritation and ability to wear the prosthesis all day.

The patient also expressed better stump and prosthesis control as compared to his previous prosthesis which was ischial load bearing. He noted a "remarkable" increase in walking distance and duration. This might be a direct benefit of the improved end-bearing allowing for elimination of ischial containment and weight transfer distally to the end-cap and the soft tissues of the thigh.

These preliminary observations support the continuous use of this technique in the setting of transfemoral amputation. Ischial liberation and restoration of end-bearing appear to improve gait, comfort, speed and distance of walking, balance, physical function and level of pain when tested at 6 months post-operatively. Furthermore, this implant offers the possibility to be used with higher levels of amputation as demonstrated by this case.

\section{Conclusion}

In conclusion, this technique reveals improvements in gait parameters, comfort and quality of life in a transfemoral amputee treated with a new procedure. The ability to transfer load through a distal end-cap and as well avoid ischial bearing appears to be responsible for the benefits noted. Regarding these favorable outcomes, larger studies and randomized control trials are required and ongoing.

\section{Ethical Clearance}

This publication is a part of a larger clinical study that was approved by Swiss Ethics (2016-01451).

\section{Conflicts of Interest}

AL declares royalties from the manufacturing company. The rest of the authors have not received grant support or research funding and they do not have any proprietary interests in the materials described in the article.

\section{References}

[1] Gritti, R. (1857) Dell' amputazione del femore al terzo inferiore e della disartictilazione del ginochhio. Annali universali di medicina Milano, 161, 5-32.

[2] Stokes, W. (1870) On Supra-Condyloid Amputation of the Thigh. Medico-Chirurgical 
Transactions, 53, 175-186. https://doi.org/10.1177/095952877005300108

[3] Taylor, B.C., et al. (2012) Gritti-Stokes Amputations in the Trauma Patient: Clinical Comparisons and Subjective Outcomes. The Journal of Bone and Joint Surgery, 94, 602-608. https://doi.org/10.2106/JBJS.K.00557

[4] Middleton, M.D. and Webster, C.U. (1962) Clinical Review of the Gritti-Stokes Amputation. British Medical Journal, 2, 574-576. https://doi.org/10.1136/bmj.2.5304.574

[5] Murakami, T. and Murray, K. (2016) Outcomes of Knee Disarticulation and the Influence of Surgical Techniques in Dysvascular Patients: A Systematic Review. Prosthetics and Orthotics International, 40, 423-435.

https://doi.org/10.1177/0309364615574163

[6] Faber, D.C. and Fielding, L.P. (2001) Gritti-Stokes (Through-Knee) Amputation: Should it Be Reintroduced? Southern Medical Journal, 94, 997-1001. https://doi.org/10.1097/00007611-200194100-00012

[7] Esquenazi, A. (2014) Gait Analysis in Lower-Limb Amputation and Prosthetic Rehabilitation. Physical Medicine and Rehabilitation Clinics of North America, 25, 153-167. https://doi.org/10.1016/j.pmr.2013.09.006

[8] Fosang, A. and Baker, R. (2006) A Method for Comparing Manual Muscle Strength Measurements with Joint Moments during Walking. Gait Posture, 24, 406-411. https://doi.org/10.1016/j.gaitpost.2005.09.015

[9] Barre, A. and Armand, S. (2014) Biomechanical ToolKit: Open-Source Framework to Visualize and Process Biomechanical Data. Computer Methods and Programs in Biomedicine, 114, 80-87. https://doi.org/10.1016/j.cmpb.2014.01.012

[10] Tinetti, M.E., Williams, T.F. and Mayewski, R. (1986) Fall Risk Index for Elderly Patients Based on Number of Chronic Disabilities. The American Journal of Medicine, 80, 429-434. https://doi.org/10.1016/0002-9343(86)90717-5

[11] Wren, T.A., et al. (2007) Gillette Gait Index as a Gait Analysis Summary Measure: Comparison with Qualitative Visual Assessments of Overall Gait. Journal of Pediatric Orthopaedics, 27, 765-768. https://doi.org/10.1097/BPO.0b013e3181558ade 